\title{
Epoxy Methacrylate Resin as Binder Polymer for Black Negative-Tone Photoresists
}

\author{
Genggongwo Shi'1, Kyeongha Baek², Seon Hong Ahn³ , Jun Bae³, Jeseob Kim¹, Lee Soon Park ${ }^{2 *}$ \\ ${ }^{1}$ CCTech. Co. Ltd., Hwaseong, South Korea \\ ${ }^{2}$ School of Material Science and Engineering, Ulsan National Institute of Science and Technology (UNIST), Ulsan, South Korea \\ ${ }^{3}$ Duksan Neolux Co., Ltd., Cheonan, South Korea \\ Email: *parkls@unist.ac.kr
}

How to cite this paper: Shi, G., Baek, K., Ahn, S.H., Bae, J., Kim, J. and Park, L.S. (2020) Epoxy Methacrylate Resin as Binder Polymer for Black Negative-Tone Photoresists. Materials Sciences and Applications, 11, 285-295.

https://doi.org/10.4236/msa.2020.115019

Received: February 17, 2020

Accepted: May 5, 2020

Published: May 8, 2020

Copyright (c) 2020 by author(s) and Scientific Research Publishing Inc. This work is licensed under the Creative Commons Attribution International License (CC BY 4.0).

http://creativecommons.org/licenses/by/4.0/

\begin{abstract}
Epoxy acrylate (EA) resin, which originates from epoxides, has long been served as a photocurable coating and adhesive material owing to its double bonds. Specifically, alkaline-developable EA resins can be used as a binder polymer in negative-tone photoresists. In this work, we synthesized a series of acidic polyester-type epoxy methacrylate resins, characterized the intermediates and products, and tested their performance as a binder polymer for the photolithographic micro-patterning of the pixel-defining layer on organic light-emitting diodes in comparison to a widely used commercial binder polymer. Copolymer-type binder polymer BP-2-2 was produced excellent patterning with no residue due to its high compatibility with the black mill base.
\end{abstract}

\section{Keywords}

Epoxy Methacrylate Resin, Negative-Tone Photoresist, Binder Polymer

\section{Introduction}

Epoxy resins, commercialized in the late 1940s, have been used in surface coatings, adhesives, laminates, and other miscellaneous fields [1]. Epoxy acrylate (EA, including epoxy methacrylate) resins can be synthesized by the first reaction of diepoxide with acrylic/methacrylic acid to give double bonds and secondary alcoholic - $\mathrm{OH}$ groups, followed by polyesterification between dianhydride and diol units. They excel epoxy resins due to their much faster radiation/thermal curing rate compared to that between curing agents and epoxy resins [2] [3] [4]. Most commercial EA resins are based on epoxy novolacs and bisphenol diglycidyl ethers because of their low cost and easy functionalization.

Apart from the well-known coating and adhesive applications, EA oligomers 
with acidic phenolic -OH [5] [6] [7] (Figure 1(a)) or carboxyl (-COOH) groups [8] [9] [10] (Figure 1(b)) have found applications as alkaline-developable binder polymer for negative-tone photoresists. These photoresists have been used for patterning color filter and black matrix [11] of liquid crystal displays (LCDs) as well as the pixel-defining layer (PDL) of organic light-emitting diodes (OLEDs) [12] [13]. Since the acidity of phenolic -OH groups of EAs based on novolac resin is relatively weak, incomplete development may occur, and the introduction of double bonds on the novolac oligomer also requires sacrificial phenolic - $\mathrm{OH}$ groups. However, EA resins with polyester linkage, as shown in Figure 1(b), have carboxylic groups as the source of acidity and have better performance in the developmental stage than that with phenolic - $\mathrm{OH}$ groups. Moreover, in the case of black photoresists, carboxyl groups can also serve as anchoring groups for the black pigment, enhancing the compatibility among other photoresist components [14].

In this study, we used dicarboxylic acids as starting materials to obtain epoxy methacrylate resin-type binder polymers and evaluated their suitability in the photolithographic process to improve black PDL patterns of OLEDs.

\section{Experimental Procedure}

\subsection{Materials}

The binder polymers were synthesized by first reacting dicarboxylic acid with glycidyl methacrylate (GMA) to form diol intermediates with methacrylate groups, followed by the reaction with dianhydrides (with/without external diol as comonomer) to obtain the final binder polymer. The chemical names, code names, and structures of diacids, dianhydrides, and diols used for the syntheses are shown in Table 1. All chemicals were reagent grades and reacted without

(a)
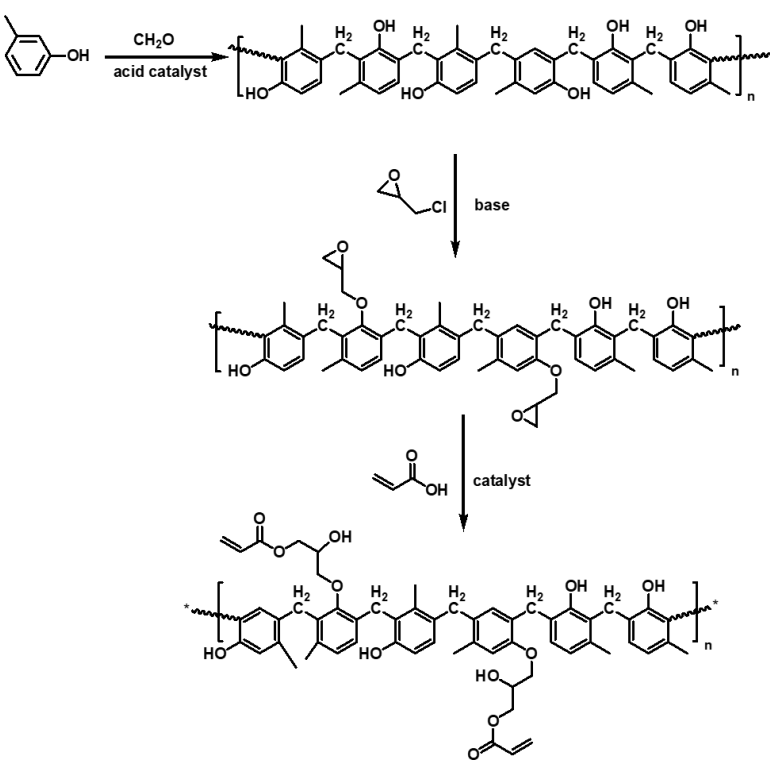

(b)
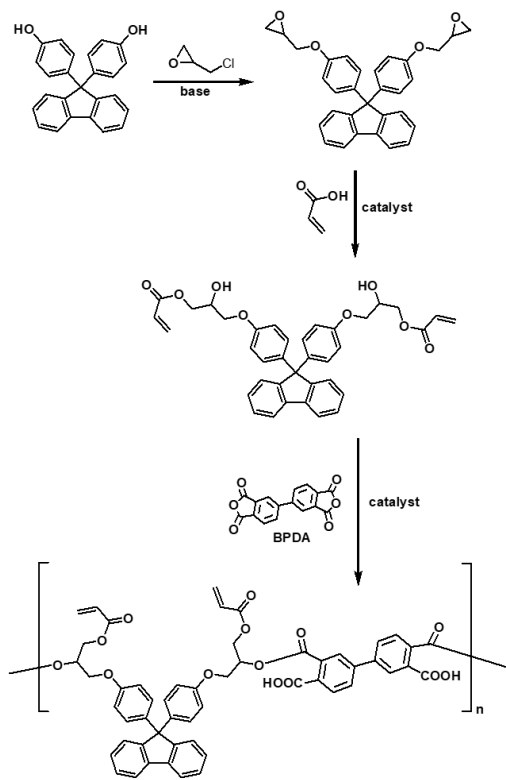

Figure 1. Representative reaction scheme of (a) cresol novolac EA resin and (b) fluorene-based polyester EA resin. 
Table 1. List of diacid, dianhydride, diol reactants, and additional selected photoresist components.

$\begin{array}{ll}\text { Code Name } & \text { Chemical Name } \\ \text { Diacids } & \text { 2,2-Bis(4-carboxyphenyl)hexafluoropropane } \\ \text { 6FDC } & \text { Itaconic Acid } \\ \text { ITCA } & \text { Methylenedisalicylic Acid (mixture of isomers) } \\ \text { 8FDC } & \text { Octafluoroadipic Acid }\end{array}$

Dianhydrides

6FDA

BPDA

4,4'-Biphthalic Anhydride (purified by sublimation)

3,9-Bis(1,1-dimethyl-2-hydroxyethyl)-2,4,8,10-tetraoxaspiro[5.5] undecane

9,9-Bis[4-(2-hydroxyethoxy)phenyl]fluorene

Bis[4-(2-hydroxyethoxy)phenyl] Sulfone

Other Species

BHT

2,6-Di-tert-butyl-4-methylphenol

TBPB

Tetrabutylphosphonium Bromide

PM6

${ }^{*}$ Acidic Multifunctional Monomer*

MSMA
3-(Trimethoxysilyl)propyl Methacrylate
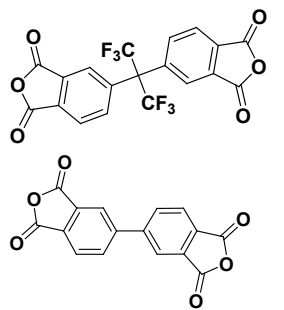

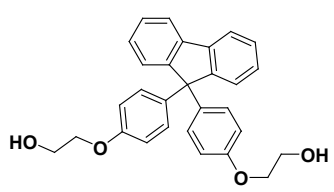

(OH
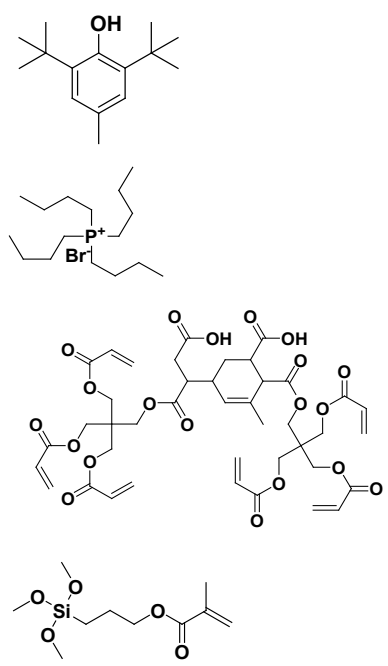
further purification. TBPB and MSMA were obtained from Alfa Aesar, PM6 was synthesized by us [10], and the other chemicals were from Tokyo Chemical Industry Co. Ltd. (TCI).

\subsection{Synthesis of Homo Binder Polymers: BP-1-x Series}

Under nitrogen atmosphere, $5 \mathrm{mmol}$ of 6FDC diacid, $10 \mathrm{mmol}$ of GMA, 0.025 mmol of polymerization inhibitor BHT, $0.05 \mathrm{mmol}$ of catalyst TBPB, and certain amount of propylene glycol methyl ether acetate (PGMEA) solvent (40 wt\% solid) were mixed and stirred at $110^{\circ} \mathrm{C}$ for $3 \mathrm{~h}$. After cooling, $5 \mathrm{mmol}$ of $6 \mathrm{FDA}$ was added with extra PGMEA (40 wt\% solid) and continued stirring at $110^{\circ} \mathrm{C}$ for another $3 \mathrm{~h}$. Up to this point, the reaction mixture appeared as a clear solution. This solution was cooled down and directly used without further work-up (BP-1-1). Binder polymers synthesized with ITCA, ODC, MLDC, and 8FDC as diacids were obtained by the same procedure, and the products were given code names as BP-1-2 BP-1-5, respectively, and those synthesized using 6FDC and BPDA were given BP-1-6 (Figure 2). Specifically, BP-1-3 was insoluble in PGMEA; therefore, it was not tested in the photolithographic evaluation.

\subsection{Synthesis of Co-Diol Type Binder Polymers: BP-2-x Series}

Under nitrogen atmosphere, $3 \mathrm{mmol}$ of 6FDC, $6 \mathrm{mmol}$ of GMA, $0.015 \mathrm{mmol}$ of inhibitor BHT, $0.05 \mathrm{mmol}$ of catalyst TBPB, and a certain amount of PGMEA solvent ( $40 \mathrm{wt} \%$ solid) were mixed and stirred at $110^{\circ} \mathrm{C}$ for $3 \mathrm{~h}$ to obtain $6 \mathrm{FG}$ diol intermediate. After cooling, $2 \mathrm{mmol}$ of external diol XDO and $5 \mathrm{mmol}$ of 6FDA were added with extra PGMEA (40 wt\% solid) and continued stirring at $110^{\circ} \mathrm{C}$ for another $3 \mathrm{~h}$. Up to this point, the reaction mixture appeared as a clear solution. This solution was cooled down and directly used without work-up (BP-2-1). Binder polymers synthesized from FDO and SDO as co-diols were coded as BP-2-2 and BP-2-3 respectively (Figure 3).
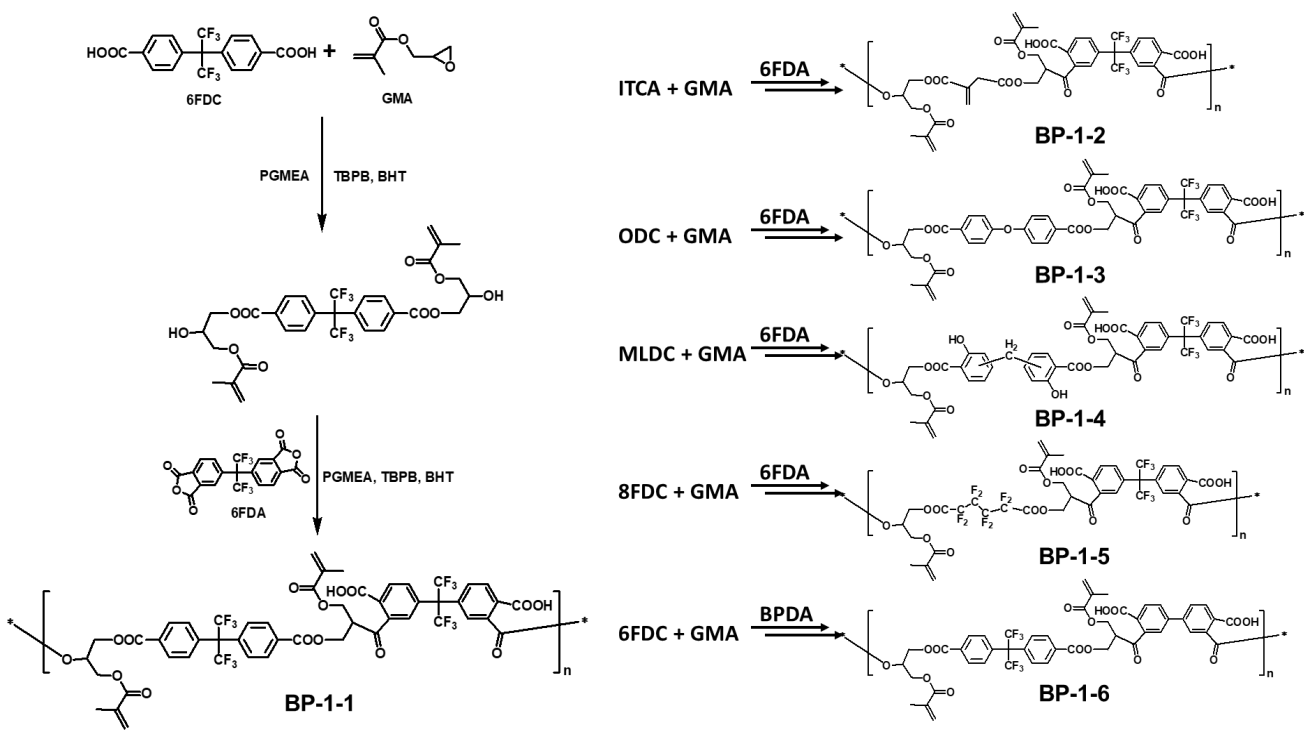

Figure 2. Synthetic scheme of representative non-cardo binder polymer BP-1-1 to BP-1-6. 


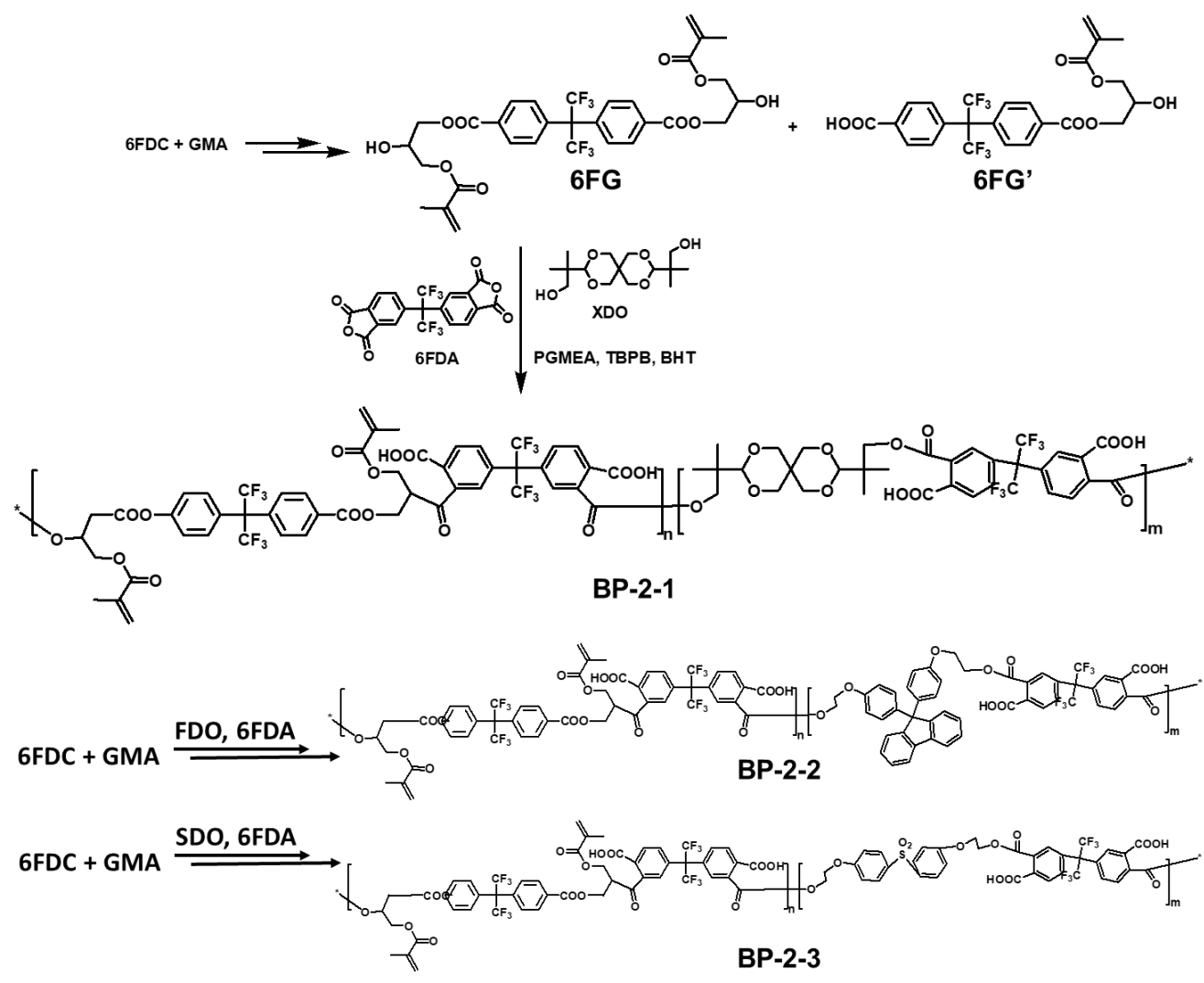

Figure 3. Synthetic scheme of copolymer-type binder polymers (BP-2-1 to BP-2-3) utilizing XDO, FDO, and SDO as comonomers.

\subsection{Characterization}

The liquid chromatography-mass spectrometry (LC-MS) was recorded using a Bruker 1200 Series \& HCT Basic System. The monomer sample in the PGMEA solution was diluted with the eluent $\left(\mathrm{MeOH}-\mathrm{H}_{2} \mathrm{O}\right.$ system) and directly subjected to the column.

The gel permeation chromatography (GPC) was recorded using an Agilent 1200 S/miniDAWN TREOS. The polymer sample in the PGMEA solution was diluted with THF and directly subjected to the column.

The Fourier-transform infrared spectroscopy (FT-IR) was recorded using a Varian 670 spectrometer. N-hexane was added to the polymer solution, and the precipitate was collected and washed 3 times with $n$-hexane using centrifugation. The resulting solid was dried under vacuum and subjected to the $\mathrm{KBr}$ pellet method.

\section{Results and Discussion}

\subsection{Syntheses and Characterizations of Binder Polymers}

Due to the low cost, high diversity, and much higher chemical stability of dicarboxylic acid species compared with diepoxies, we utilized various diacids as starting materials to react with monoepoxide GMA to obtain diepoxy methacrylate-type diol monomers. As shown in Figure 1(b), to get EA diol monomer 
from diepoxide as a starting material, acrylic acid increases the cost compared with that synthesized using dicarboxylic acid reacted with glycidyl methacrylate (GMA). Since the reactivity between the epoxide group and the carboxyl group is higher than that between the epoxide group and the secondary - $\mathrm{OH}$ group under non-basic catalytic conditions [15], the ring-open polymerization could be suppressed during the formation of diepoxy methacrylate monomers in the presence of TBPB.

To confirm the formation of the 6FG intermediate, its solution starting from 6FDC and GMA (6FG and 6FG', represented as $\mathrm{M}$ and $\mathrm{M}^{\prime}$ in the LC-MS graph) was analyzed with LC-MS. Between 16.0 and 16.4 min of retention time, major signals of $6 \mathrm{FG}\left([\mathrm{M}+\mathrm{Na}]^{+}\right.$and $\left.[\mathrm{M}-\mathrm{OH}]^{+}\right)$and minor signal of $\left[\mathrm{M}^{\prime}-\mathrm{OH}\right]^{+}$ were found in which the extraction of hydroxyl radical could be caused by the cleavage of the secondary $\mathrm{C}-\mathrm{O}$ bond in a glycerol group under the mass spectrometric detector (Figure 4).

The molecular weights of all binder polymers were in the range of 2000 and 4000 , in which the copolymers showed a little higher $\mathrm{Mw}$, probably due to the bulkiness of the co-diols XDO, FDO, and SDO (Figure 5). So, each binder polymer or oligomer may have 3 - 5 repeating units on average, providing it with suitable viscosity and reactivity toward cross-linking by free radical polymerization mechanism.

The FT-IR diagrams (Figure 6) of BP-1-1 and BP-2-3 clearly exhibited the $\mathrm{C}=\mathrm{O}$ stretching of aryl ester groups $\left(1732 \mathrm{~cm}^{-1}\right)$, while the $\mathrm{C}-\mathrm{F}$ stretching signals (1000 - $1400 \mathrm{~cm}^{-1}$ ) of BP-1-1 were much higher than those of BP-2-3 due to the higher occupation of 6FDC units of BP-1-1 [16].

\subsection{Photolithographic Evaluation of Binder Polymers}

The photolithographic performance of the synthesized epoxy methacrylate binder polymers was evaluated in comparison to the commercial cardo binder polymer CBP, whose structure is shown in Figure 1(b). In a typical black photoresist formulation (Table 2), $22 \mathrm{wt} \%$ binder polymer and $6 \mathrm{wt} \%$ acidic multifunctional monomer (PM6, $\mathrm{f}=6$ ) were used to provide double bonds for the photo-crosslinking reaction. The MSMA adhesion promotor (1 wt\%) was added to enhance the adhesion of the black PDL pattern to the silicon wafer substrate by the chemical reaction between the MSMA siloxane and hydroxyl silicon on the wafer surface. In this work, the UV dose was fixed as $60 \mathrm{~mJ} / \mathrm{cm}^{2}$.

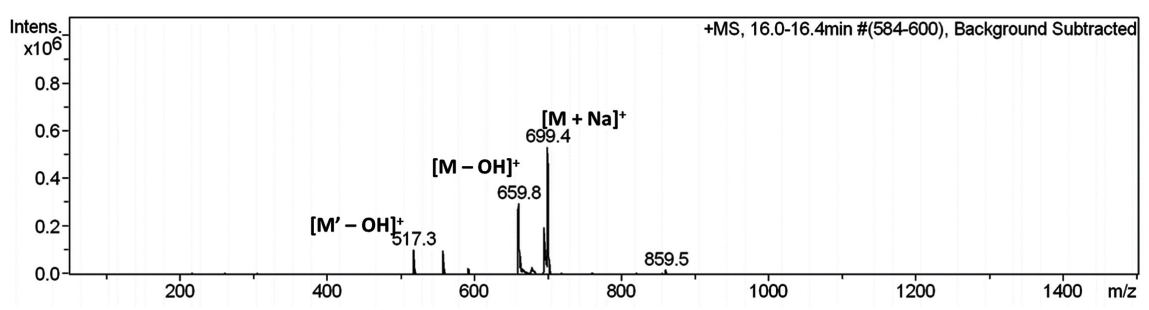

Figure 4. LC-MS graph of 6 FG at 16.0-16.4 min of retention time. 

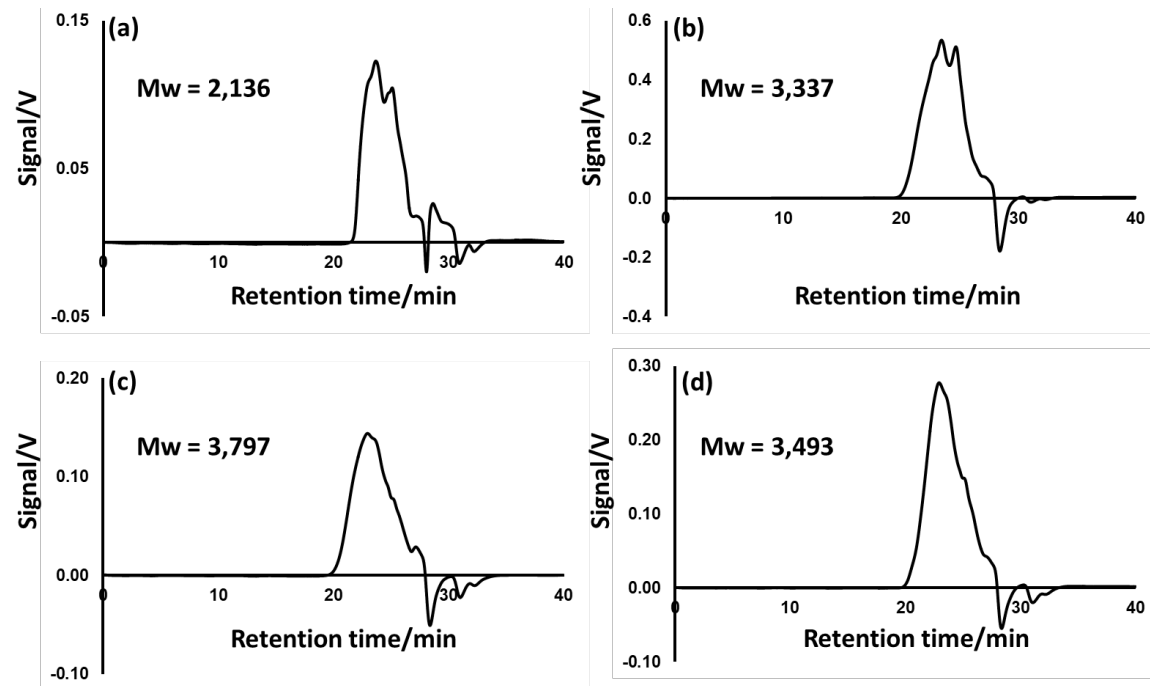

Figure 5. GPC graphs of selective binder polymers: (a) BP-1-1, (b) BP-1-6, (c) BP-2-1, and (d) BP-2-2 binder polymers.

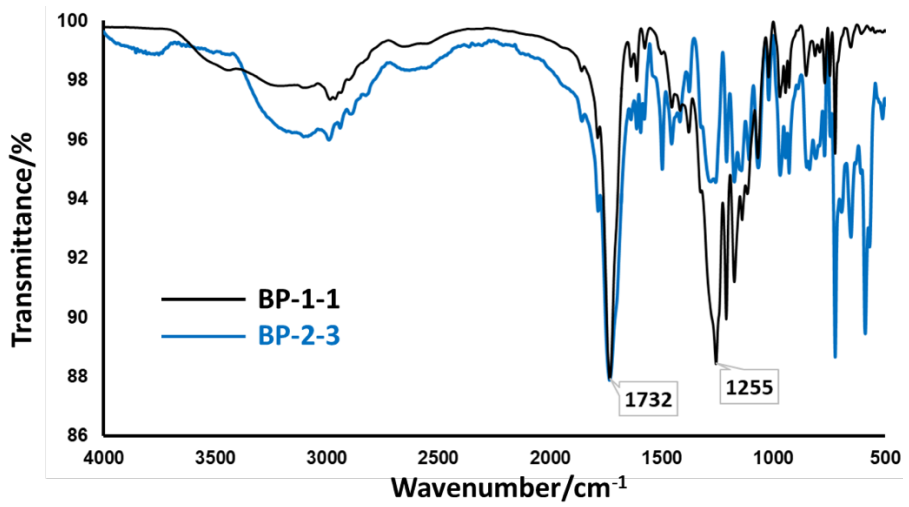

Figure 6. FT-IR diagrams of selective binder polymers: BP-1-1 and BP-2-3.

Table 2. Black photoresist formulations using CBP as standard binder polymer and other epoxy acrylate binder polymers.

\begin{tabular}{|c|c|c|c|}
\hline \multicolumn{2}{|c|}{ Photoresist code } & PR-0 & PR-x-y \\
\hline Photo-initiator & SPI-03 (Samyang. Co. Ltd.) & 4.0 & 4.0 \\
\hline Photo-sensitizer & Darocure ITX (Ciba, Switz) & 1.0 & 1.0 \\
\hline Binder polymer (45 wt $\%$ ) & CBP (KISCO, Korea) & 20 & \\
\hline Binder polymer (40 wt $\%$ ) & BP-x-y (BP-1-1 6, BP-2-1 3) & & 22 \\
\hline $\begin{array}{l}\text { Multifunctional monomer } \\
\qquad(50 \mathrm{wt} \%)\end{array}$ & PM6 & 6.0 & 6.0 \\
\hline Adhesion promotor & MSMA & 1.0 & 1.0 \\
\hline Solvent & PGMEA & 12 & 10 \\
\hline Black pigment & BK-4926 (Tokushiki, Japan) & 56 & 56 \\
\hline Total & wt $\%$ & 100 & 100 \\
\hline
\end{tabular}


The black photoresist with CBP binder polymer (PR-0) gave better pattern and residue level (Table 3) under $60 \mathrm{~mJ} / \mathrm{cm}^{2}$ of UV dose and $4 \mathrm{wt} \%$ photo-initiator compared with our previous studies [9] [10]. Among the 5 soluble homopolymer candidates, BP-1-2 and BP-1-4 caused issues during development with aqueous alkaline, presumably due to the high double bond density (mmol of double bonds in $1 \mathrm{~g}$ of sample, DBD) and high adhesion to the substrate, respectively. When the linear diacid $8 \mathrm{FDC}$ was used as the starting material, the photo-patterning and residue became better (PR-1-5) due to the higher number of $-\mathrm{CF}_{2}$ - groups in the binder polymer, which enhanced the compatibility with the black mill base. However, the black photoresist (PR-1-1) with BP-1-1 as the binder polymer exhibited better performance in the photolithographic evaluation (Figure 7(b)) than PR-0 (Figure 7(a)) since the binder polymer BP-1-1 had $-\mathrm{CF}_{3}$ groups in both diacid (6FDC) and dianhydride (6FDA). The performance of PR-1-6 was not as good as PR-1-1, probably due to the lack of $-\mathrm{CF}_{3}$ groups in BPDA dianhydride instead of 6FDA.

To further reduce the residue on the substrate after development, we utilized 3 comonomer diols, as shown in Figure 3 to synthesize copolymer-type binder polymers (PR-2-1, PR-2-2, PR-2-3). Among the three copolymer-type binder polymers, the binder polymer BP-2-2 gave the best photolithographic patterning, as shown in Table 4 and Figure 7(d). The BP-2-1 binder polymer made with XDO comonomer showed slightly over-crosslinked pattern (Figure $7(\mathrm{c})$ ), while the BP-2-3 binder polymer gave no pattern. This could be explained by the steric effect of co-diols: FDO offers planar fluorene structure outside the polymer chain, which could more effectively interact with the black mill base, and good compatibility with $-\mathrm{CF}_{3}$ groups.

Table 3. List of epoxy methacrylate binder polymer properties and photolithographic performances.

\begin{tabular}{ccccccc}
\hline Performance & PR-0 & PR-1-1 & PR-1-2 & PR-1-4 & PR-1-5 & PR-1-6 \\
\hline Binder polymer & CBP & BP-1-1 & BP-1-2 & BP-1-4 & BP-1-5 & BP-1-6 \\
\hline Diacid & & 6FDC & ITCA & MLDC & 8FDC & 6FDC \\
dianhydride & & 6FDA & 6FDA & 6FDA & 6FDA & BPDA \\
$\begin{array}{c}\text { Monomers } \\
\text { Pattern Evaluation }\end{array}$ & 2 & 1 & 4 & 4 & 3 & 3 \\
$\begin{array}{c}\text { DBD (mmol/g) } \\
\text { (Good-bad, 1-5) }\end{array}$ & 2.22 & 1.81 & 3.56 & 1.94 & 1.99 & 2.09 \\
$\begin{array}{c}\text { Residue Evaluation } \\
(\text { Good-bad, 1-5) }\end{array}$ & 2 & 2 & 5 & 5 & 3 & 3 \\
\hline
\end{tabular}

${ }^{a} 1$ : The pattern shapes and sizes are almost the same as those of the photomask. 2: The pattern shapes are almost the same as those of the photomask, but the sizes are within $90 \%$ error. 3: The pattern shapes appear a little distorted or wavy. 4: The patterns lost thickness or are incomplete. 5: There are almost no patterns at all. ${ }^{\mathrm{b}}$ 1: The developed parts are trace-free and appear like new wafer under OM. 2: The developed parts show traces under OM. 3: The developed parts show traces to the naked eye. 4: The developed parts show obvious residues to the naked eye. 5: Incomplete development. 
Table 4. List of black photoresists containing copolymer-type binder polymer and their photolithographic performances.

\begin{tabular}{ccccc}
\hline Performance & PR-0 & PR-2-1 & PR-2-2 & PR-2-3 \\
\hline Binder polymer & CPB & BP-2-1 & BP-2-2 & BP-2-3 \\
\hline $\begin{array}{c}\text { Co-monomer diols } \\
\text { Pattern Evaluation } \\
\text { (Good-bad, 1-5) } \\
\text { DBD (mmol/g) } \\
\begin{array}{c}\text { Residue Evaluation } \\
\text { (Good-bad, 1-5) }\end{array}\end{array} \quad 2 \quad$ XDO & FDO & SDO \\
\hline
\end{tabular}

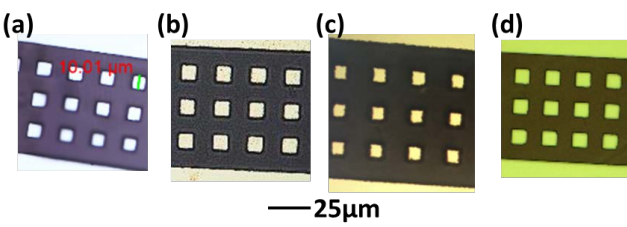

Figure 7. Representative optical microscope images of black PDL patterns obtained with (a)

PR-0, (b) PR-1-1, (c) PR-2-1, and (d) PR-2-2.

\section{Conclusion}

In this work, a series of epoxy methacrylate-type binder polymers were synthesized using dicarboxylic acids as starting materials. The photo-initiator loading, UV dose, and DBD of binder polymers contributed to the photolithographic cross-linking reaction simultaneously; therefore, we tuned these parameters carefully to optimize cross-linking. Moreover, $-\mathrm{CF}_{3}$ groups and bulky fluorene groups were found to improve the compatibility with the black mill base, resulting in less residue on the wafer after the development. The black photoresist PR-2-2 with the copolymer-type binder polymer BP-2-2 produced the optimal PDL pattern with the least residue in the photolithographic tests. This work can pave the way for the design of binder polymers for negative-tone black photoresist and the optimization of photolithographic process conditions.

\section{Acknowledgements}

This work was supported by the Technology Innovation Program (or Industrial Strategic Technology Development Program; 10063289, Development of High-Temperature Negative-Tone Photosensitive Black Resin and Fabrication Process for Pol-less AMOLED Devices) funded by the Ministry of Trade, Industry \& Energy, Korea.

\section{Conflicts of Interest}

The authors declare no conflicts of interest regarding the publication of this paper. 


\section{References}

[1] May, C.A. (1988) Epoxy Resins: Chemistry and Technology, Second Edition.

[2] Chattopadhyay, D.K., Panda, S.S. and Raju, K.V.S.N. (2005) Thermal and Mechanical Properties of Epoxy Acrylate/Methacrylates UV Cured Coatings. Progress in Organic Coatings, 54, 10-19. https://doi.org/10.1016/j.porgcoat.2004.12.007

[3] Hong, B.T., Shin, K.S. and Kim, D.S. (2005) Ultraviolet-Curing Behavior of an Epoxy Acrylate Resin System. Journal of Applied Polymer Science, 98, 1180-1185. https://doi.org/10.1002/app.22130

[4] Park, Y.J., Lim, D.H., Kim, H.J., Park, D.S. and Sung, I.K. (2009) UV- and Thermal-Curing Behaviors of Dual-Curable Adhesives Based on Epoxy Acrylate Oligomers. International Journal of Adhesion and Adhesives, 29, 710-717. https://doi.org/10.1016/j.ijadhadh.2009.02.001

[5] Tsukada, K., Isobe, A., Hayashi, N., Abo, M. and Ogawa, K. (1976) Photosensitive Epoxy-Acrylate Resin Compositions. US Patent No. US3989610A.

[6] Hosomi, T., Hayai, H. and Baba, T. (1998) Resin Composition and Printed Circuit Board Using the Same. US Patent No. US5726219A.

[7] Shi, G., Kim, J., Baek K., Bae, J. and Park, L.S. (2019) Melt and One-Pot Solution Synthesis of Thermally Stable, Organosoluble and Photocurable Acidic Polyimides. Materials Sciences and Applications, 10, 687-696. https://doi.org/10.4236/msa.2019.1011049

[8] Kim, J., Park, K., Lee, D. and Bae, J.Y. (2011) Synthesis of Fluorene-Containing Photosensitive Polymer and Its Application to the Carbon Black-Based Photoresist for LCD Color-Filter. Polymer (Korea), 35, 87-93. https://doi.org/10.7317/pk.2011.35.1.87

[9] Shi, G., Park, S.H., Kim, J., Kim, M. and Park, L.S. (2018) Side-Chain Polyimides as Binder Polymers for Photolithographic Patterning of a Black Pixel Define Layer for Organic Light Emitting Diode. International Journal of Polymer Science, 2018, Article ID:3790834. https://doi.org/10.1155/2018/3790834

[10] Shi, G., Baek, K., Ahn, S.H., Bae, J., Kim, J. and Park, L.S. (2019) Synthesis of New Binder Polymers for Photolithographic Patterning of Black Pixel Define Layers of Organic Light Emitting Diode. Materials Sciences and Applications, 10, 687-696. https://doi.org/10.4236/msa.2019.1011049

[11] Kuo, K.H., Chiu, W.Y., Hsieh, K.H. and Don, T.M. (2009) Novel UV-Curable and Alkali-Soluble Resins for Light-Shielding Black Matrix Application. European Polymer Journal, 45, 474-484. https://doi.org/10.1016/j.eurpolymj.2008.10.044

[12] Diethelm, M., Penninck, L., Altazin, S., Hiestand, R., Kirsch, C. and Ruhstaller, B. (2018) Quantitative Analysis of Pixel Crosstalk in AMOLED Displays. Journal of Information Display, 19, 61-69. https://doi.org/10.1080/15980316.2018.1428232

[13] Kim, N. S., Lee, W.Y. and Suh, M.C. (2018) Suppression of the Color Shift of Microcavity Organic Light-Emitting Diodes Through the Introduction of a Circular Polarizer with a Nanoporous Polymer Film. Journal of Information Display, 19, 91-98. https://doi.org/10.1080/15980316.2018.1454348

[14] Kuo, K.H., Chiu, W.Y. and Don, T.M. (2009) Synthesis of UV-Curable/Alkali-Soluble Dispersants Used for Black Photoresist with a High Loading of Carbon Black. Journal of Applied Polymer Science, 115, 1803-1813. https://doi.org/10.1002/app.31304

[15] Dass, C. (2006) Fundamentals of Contemporary Mass Spectrometry. https://doi.org/10.1002/0470118490

[16] Park, H., Yoo, S., Ahn, H., Bang, J., Jeong, Y., Yi, M., Won, J.C., Jung, S. and Kim, 
Y.H. (2019) Low-Temperature Solution-Processed Soluble Polyimide Gate Dielectrics: From Molecular-Level Design to Electrically Stable and Flexible Organic Transistors. ACS Applied Materials \& Interfaces, 11, 45949-45958.

https://doi.org/10.1021/acsami.9b14041 